\title{
Transcatheter arterial embolization of malignant pelvic solitary fibrous tumor: case report and literature review
}

\author{
Xingjian Yan ${ }^{1 \#}$, Chao Zheng ${ }^{2 \#}$, Jin Wang $^{1}$, Dawei Li ${ }^{1}$, Ji Lu$^{1}$, Liang He ${ }^{1 \wedge}$, Chunxi Wang ${ }^{1}$ \\ ${ }^{1}$ Department of Urology, The First Hospital of Jilin University, Changchun, China; ${ }^{2}$ Department of Neurology and Neuroscience Center, First \\ Hospital of Jilin University, Changchun, China \\ "These authors contributed equally to this work. \\ Correspondence to: Chunxi Wang; Liang He. Department of Urology, The First Hospital of Jilin University, 71 Xinmin Road, Chaoyang District, \\ Changchun 130021, China. Email: chunxi@jlu.edu.cn; lianghe9278@jlu.edu.cn.
}

\begin{abstract}
Pelvic malignant solitary fibrous tumor (SFT) is a relatively rare disease, and literature on radical resection with transcatheter arterial embolization of pelvic SFT is lacking. In this work, we report on a 55-year-old man with a presacral mass who was hospitalized at our department. Computed tomography and magnetic resonance imaging indicated pelvic space-occupying lesions that were $12 \mathrm{~cm} \times 10 \mathrm{~cm}$ in size and pelvic lesions that were not clearly demarcated from the right posterior wall of the bladder and the right ureter. This result suggested severe secondary hydronephrosis of the right renal pelvis. The patient underwent transcatheter iliac arterial embolization. Radical tumor resection was performed, and the results of pathological examination confirmed the diagnosis of malignant pelvic SFT. There was no SFT recurrence in this patient at 1-year follow-up. Herein, we report on the treatment of a patient with malignant pelvic SFT, a rare condition, who underwent successful radical resection after transcatheter arterial embolization. Transcatheter arterial embolization can block the blood supply of the SFT as much as possible and improve the possibility of tumor resection. In the future, pelvic SFTs can be considered improving the resection rate by transcatheter arterial embolization before surgery.
\end{abstract}

Keywords: Malignant solitary fibrous tumor; transcatheter arterial embolization; case report.

Submitted May 22, 2021. Accepted for publication Oct 20, 2021.

doi: $10.21037 /$ tcr-21-887

View this article at: https://dx.doi.org/10.21037/tcr-21-887

\section{Introduction}

Solitary fibrous tumor (SFT) is a rare mesenchymal tumor mainly reported in the pleura (1). Recently, an increasing number of extrapleural SFTs, including pelvic SFTs, have been found in the clinical setting. Pelvic SFTs can be either benign or malignant. Malignant pelvic SFTs are extremely rare. The clinical diagnosis and treatment of malignant pelvic SFTs are sophisticated. Herein, we report on a case with malignant pelvic SFT and systematically reviewed the literature on pelvic SFTs. From our case, we put forward novel clinical experiment that transcatheter arterial embolization of malignant pelvic SFT could improve the tumor resection rate and prognosis. We present the following article in accordance with the CARE reporting checklist (available at https://dx.doi.org/10.21037/tcr-21-887).

\section{Case presentation}

A 55-year-old man presented to our hospital with persistent lower abdominal pain. The patient was otherwise healthy, with no family history of malignant tumors. Laboratory examination results were normal. Computed tomography

\footnotetext{
^ ORCID: 0000-0003-0966-0526.
} 

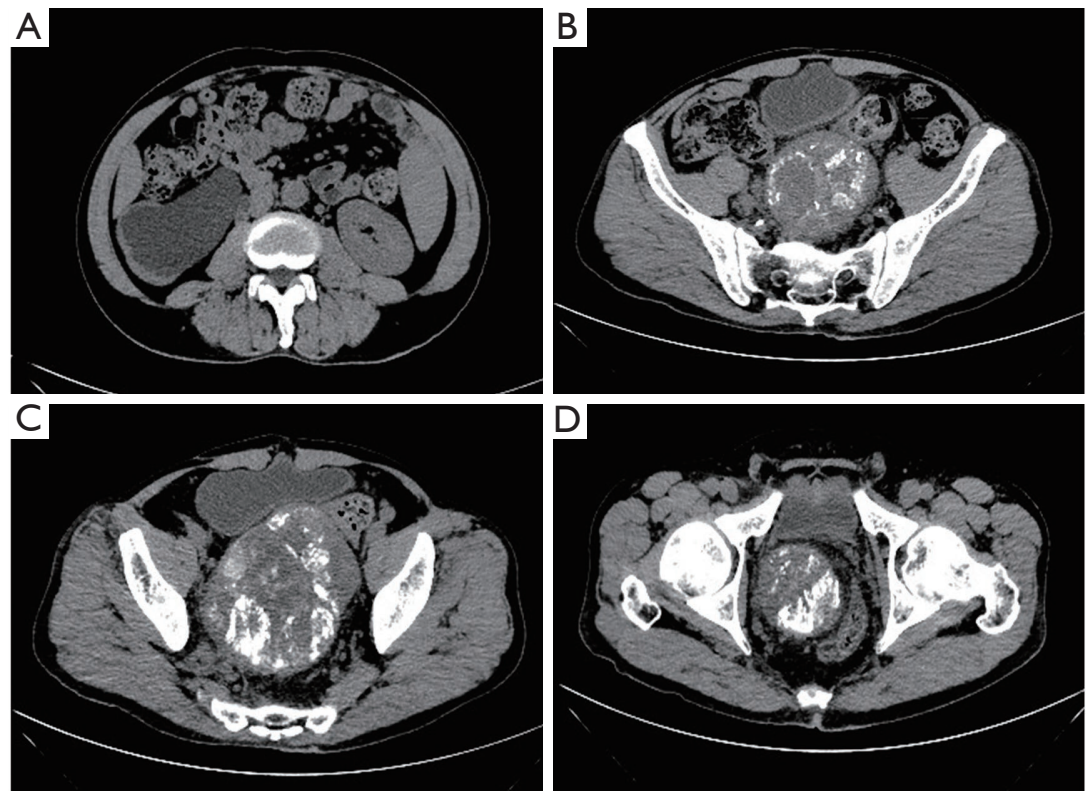

Figure 1 The CT examination results of different planes. (A) The sign of right hydronephrosis. (B-D) Different layers of the pelvic SFT. CT; computed tomography; SFT, solitary fibrous tumor.
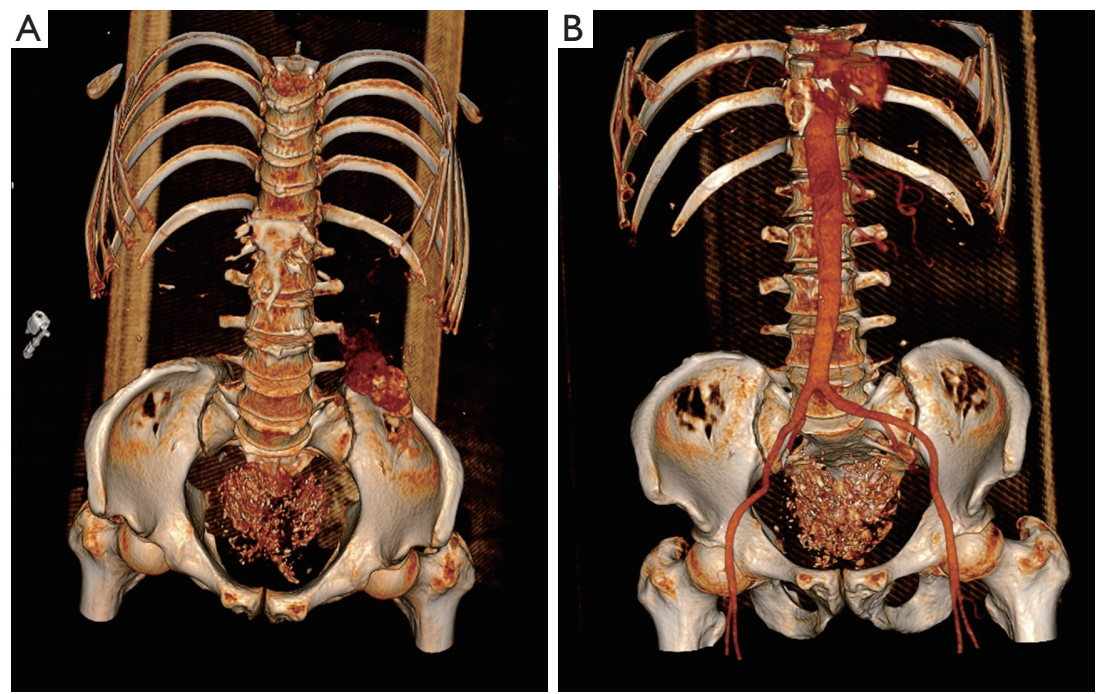

Figure 2 The three-dimensional reconstruction of the enhanced CT. (A) The CT three-dimensional reconstruction of pelvic SFT. (B) The CT three-dimensional reconstruction of pelvic SFT arteries. CT; computed tomography; SFT, solitary fibrous tumor.

(CT) and magnetic resonance imaging (MRI) indicated significant necrosis and calcification in the tumor.

Abdominal enhancement CT results indicated that there was a large mass of abnormal density behind the bladder. The pelvic mass was about $12.4 \mathrm{~cm} \times 10.1 \mathrm{~cm}$ in size, and the CT value was $40 \mathrm{HU}$. There were multiple calcifications in the pelvic mass. Enhanced CT examination results showed slight enhanced density at the edge of the lesion (Figure 1). The 3D reconstruction of the enhanced CT is shown in Figure 2.

Pelvic MRI indicated significant abnormal signal shadows between the posterior bladder and rectum, about $12.4 \mathrm{~cm}$ $\times 9.1 \mathrm{~cm} \times 8.1 \mathrm{~cm}$ in size (Figure 3). Inconsistent signals 

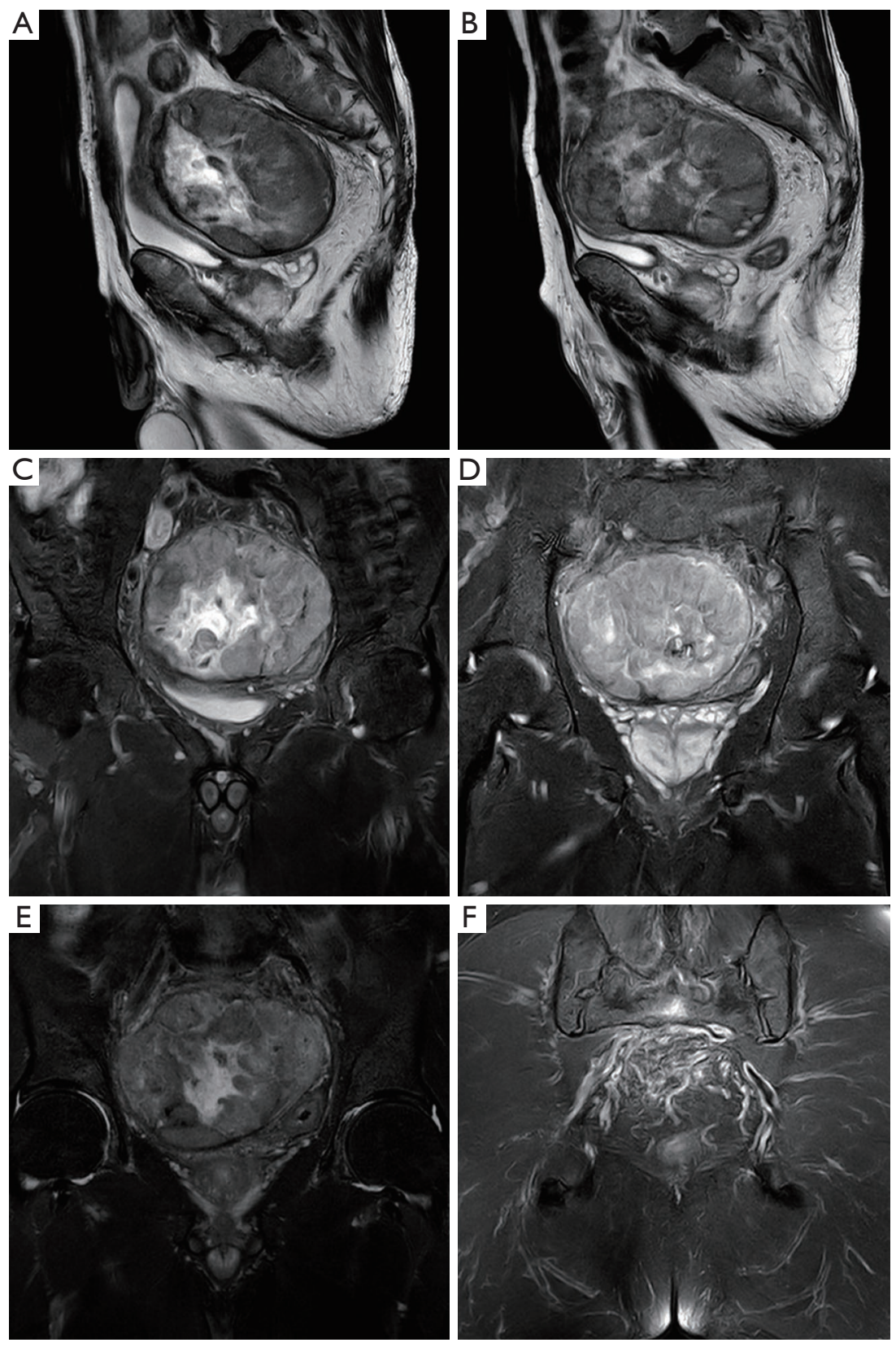

Figure 3 The MRI examination results of different planes and angles. (A,B) Sagittal plane MRI figure. (C-F) Different planes of pelvic posterior coronal MRI figure. MRI, magnetic resonance imaging.

were seen on long $\mathrm{T} 1$ and $\mathrm{T} 2$ images, and short $\mathrm{T} 1$ and $\mathrm{T} 2$ signals were seen in the lesion. A linear low-signal envelope could also be seen around the lesion. The lesion showed markedly uneven enhancement on enhanced examination. The edge of the lesion was also significantly enhanced. The boundary between the lesion and the right ureter was unclear, and the right ureter was obviously expanded. No obvious enlarged lymph node was found in the pelvic cavity.

The patient underwent transcatheter iliac arterial embolization, which obstructed the blood supply of the tumor. Tumor resection was performed, and the malignant pelvic SFT was completely removed within 1 hour.

The tumor envelope was intact, with some necrosis and calcification inside (Figure 4A). Hematoxylin and eosin 

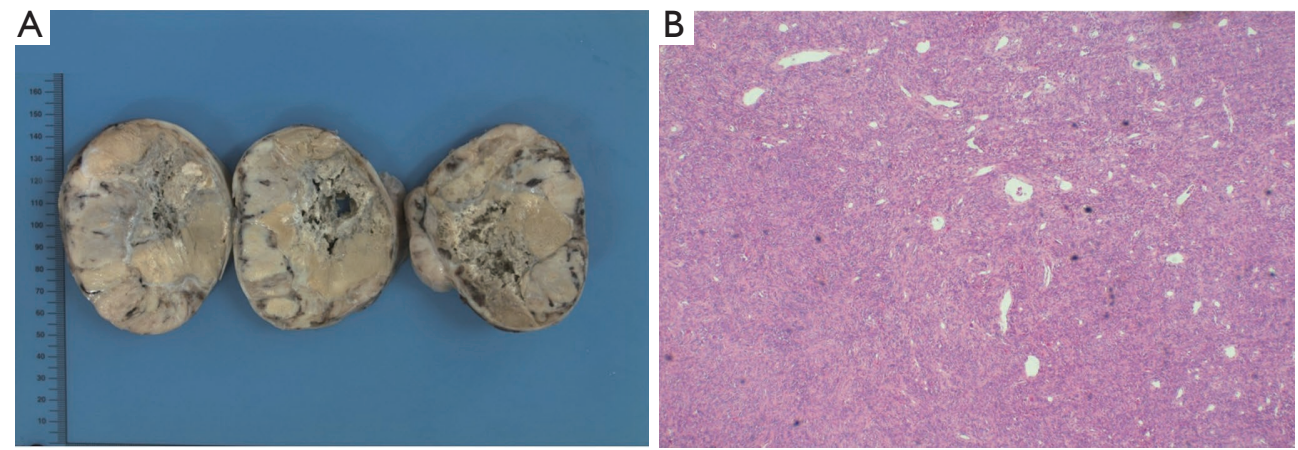

Figure 4 The general picture of SFT and H\&E picture. (A) The photo of pelvic SFT of the patient. (B) The H\&E result of pelvic SFT. Magnificent 200×. SFT, solitary fibrous tumor.

staining confirmed that that pelvic tumor was a malignant SFT with significant infarction (Figure $4 B$ ). As shown in Figure 5, the immunohistochemical results exhibited vimentin (-), cytokeratin (CK)-pan (focal positive), CD34 (+), CD99 (+), S-100 (-), B-cell lymphoma-2 (Bcl-2) (+), Ki67 (+10\%), P53 (+20\%), CD31 (-), smooth muscle actin (-), h-Caldesmon (-), desmin (-), CD117 (-), Dog-1 (-), ALK $(-)$, and STAT6 (+). Immunohistochemical results further confirmed the diagnosis of malignant pelvic SFT. The patient recovered well after the surgery and did not require adjuvant therapy. The patient did not accept postoperative radiotherapy or chemotherapy. The patient was followed up at 1 year, and had no recurrence of malignant SFT or lower abdominal pain symptoms. The patient was able to perform activities of daily living normally without discomfort.

All procedures performed in studies involving human participants were in accordance with the ethical standards of the ethics committee of the First Hospital of Jilin University and with the Helsinki Declaration (as revised in 2013). Written informed consent was obtained from the patient for publication of this case report and accompanying images.

\section{Discussion}

SFT, a rare mesenchymal tumor (2), was first reported in 1931 by Klemperer and Rabin (3). It was first described as a benign variant of mesothelioma (4). Although most SFTs are benign, about $20 \%$ of SFTs are malignant. The 2013 World Health Organization (WHO) classification of soft tumor defines SFTs as hypercellular, mitotically active tumors [ $>4$ mitosis/10 high-power fields (HPF)], with cytological atypia, tumor necrosis, and/or infiltrative margins (5). The most effective treatment for SFT is complete resection. Furthermore, postoperative adjuvant chemotherapy for malignant SFT could prevent recurrence and metastasis.

The SFT can also exhibit various forms, including osteosarcomatous and chondrosarcomatous components (6), which makes the diagnosis of SFT challenging. Radiographic diagnosis is mainly dependent on CT and MRI. The CT values and MRI signal intensity of SFTs vary depending on the amount of collagen, vascular tissue, and myxoid and cystic degeneration in the tumor (7). Different types of SFTs exhibit varying manifestations on CT and MRI. However, Li et al. concluded that there was no difference in CT and MRI findings between malignant and benign SFTs (8); therefore, CT and MRI cannot be used to diagnose SFT. Fluorodeoxyglucose positron emission tomography (FDG-PET) can also be used as a supplementary examination for malignant or benign SFT. In previous cases, malignant SFTs showed increased FDG uptake, while the uptake of benign SFTs was negative (9). The clinical behavior of SFTs is hard to predict. Therefore, many SFTs are discovered after surgical treatment (10). Postoperative pathological diagnosis is the most reliable method for distinguishing SFT from other mesenchymal tumors and is the gold standard for the diagnosis for SFT. As a rare mesenchymal tumor, SFT exhibits as "patternless" ovoid and spindle cells, while it often has a unique immunohistochemistry staining results (positive for STAT6, CD34, CD99, and Bcl-2) (9). CD34, CD99, vimentin, and Bcl-2 constitute the basic pathological diagnosis of SFT (7), and most SFTs are positive for CD34, CD99, and Bcl-2 (2). The rearrangement of NGFI-A binding protein 2 (NAB2)STAT6 can be found in almost all SFTs, which leads to 

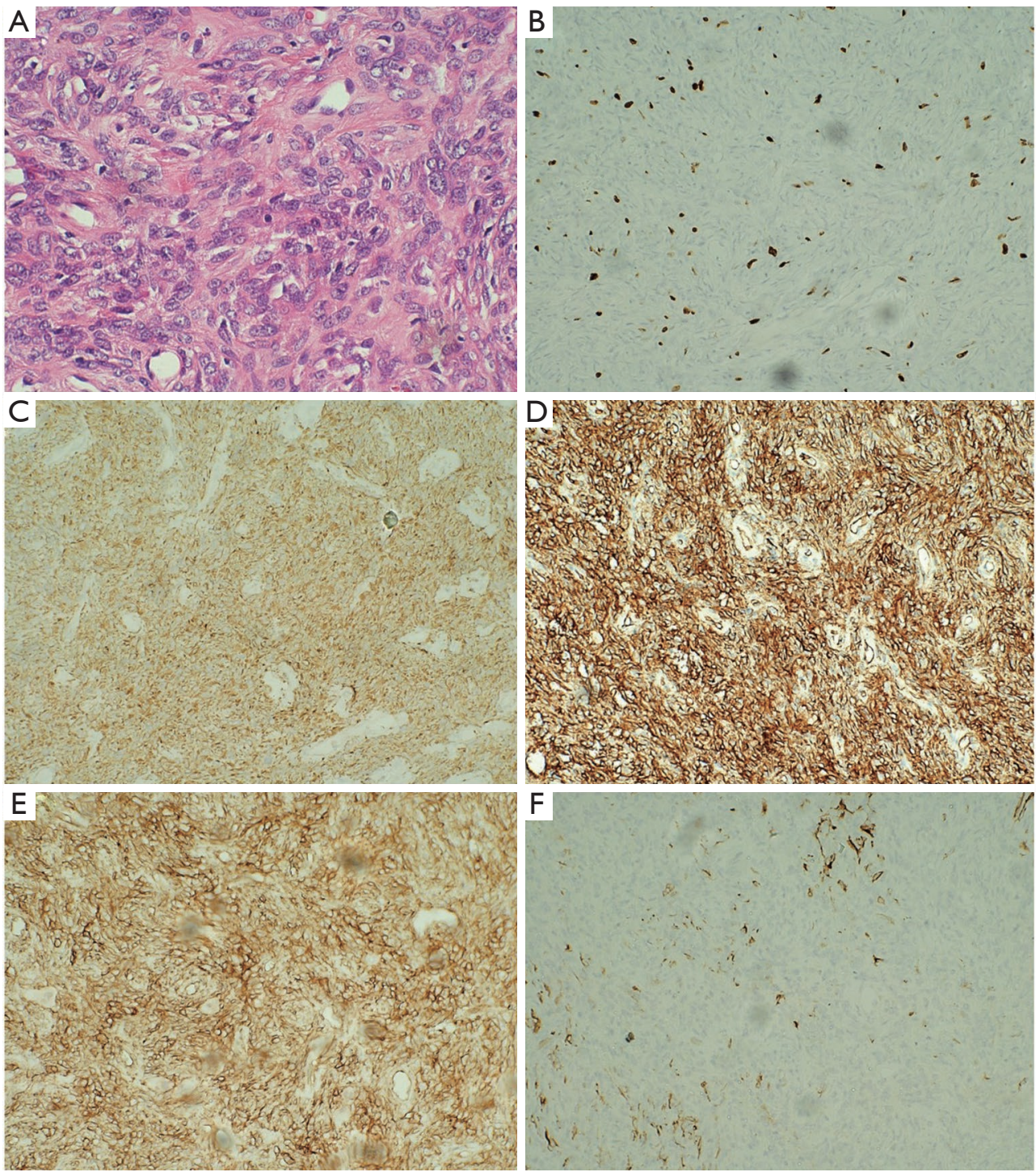

Figure 5 The immunohistochemical results. (A) H\&E result of pelvic SFT; (B) Ki-67 result; (C) Bcl result; (D) CD34 result; (E) CD99; (F) CK-pan result. Magnificent 200×. SFT, solitary fibrous tumor.

the positive expression of STAT6 (11). STAT6 detection can increase the accuracy of SFT diagnosis. Currently, the rearrangement of NAB-STAT6 is considered as specific histological diagnostic standard for SFT. SFTs can undergo dedifferentiation transformation, and this particular type of SFT is difficult to distinguish from Ewing's sarcoma, therefore making SFT diagnosis difficult (12). Awareness of immunohistochemical and molecular biological characteristics can help to accurately diagnose SFT.

Malignant SFTs are rare, and there are no established criteria to diagnosis malignant SFTs (13). In their study,
England et al. concluded the criteria for malignant SFTs (14). As shown in Table 1, malignant SFTs have the following characteristics: (I) hypercellularity ( $>4$ mitoses $/ 10$ HPF), (II) nuclear pleomorphism, (III) necrosis, (IV) tumor infiltrative growth, and (V) tumor size $>10 \mathrm{~cm}$. Although there are obvious differences between benign and malignant SFTs, some SFTs are incorrectly characterized as benign. Retrospective studies have shown that patients with benign pathological SFTs may also have recurrence after resection (5). This can be attributed to the limitations of pathological diagnosis. For indistinguishable SFTs, total 
Table 1 The systematic review of pelvic SFT case reports

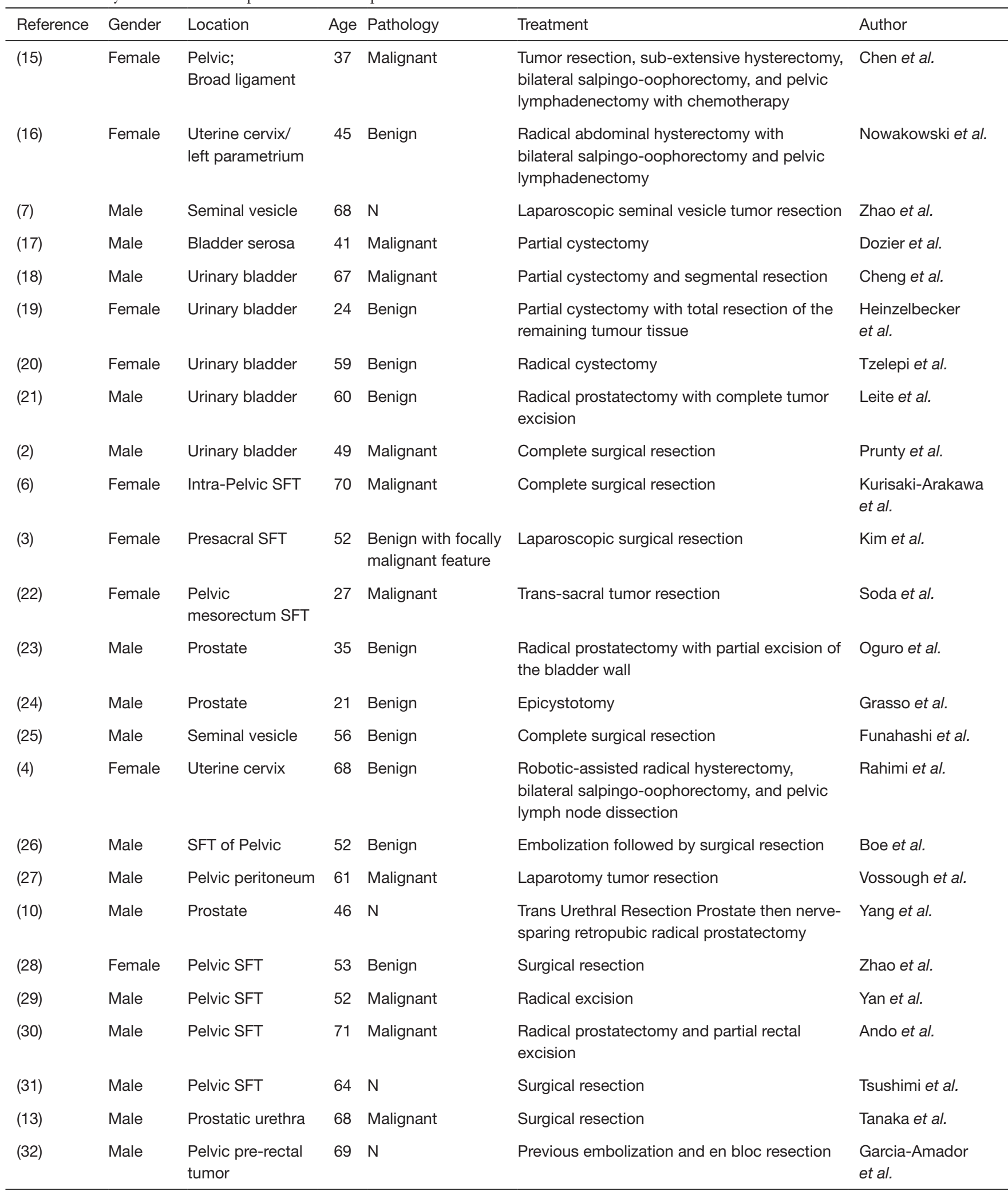

Table 1 (continued) 
Table 1 (continued)

\begin{tabular}{|c|c|c|c|c|c|c|}
\hline Reference & Gender & Location & Age & Pathology & Treatment & Author \\
\hline (34) & Female & Perianal tumor & 56 & Benign & Trans-sacral tumor resection & Katsuno et al. \\
\hline (35) & Male & Pelvic tumor & 52 & Malignant & Pelvic tumor resection & Gao et al. \\
\hline
\end{tabular}

$\mathrm{N}$ means not mentioned. SFT, solitary fibrous tumor.

Table 2 Criteria for malignant pelvic SFT

Malignant SFT characteristic
1. Presence of hypercellularity ( $>4$ mitoses $/ 10 \mathrm{HPF})$
2. Nuclear pleomorphism
3. Necrosis
4. Tumor infiltrative growth
5. Tumor size $>10 \mathrm{~cm}$

SFT, solitary fibrous tumor; HPF, high-power fields.

resection is necessary. Therefore, detailed preoperative preparation and complete resection of tumors are the best treatments for SFT.

SFT mainly occurs in the pleura. In their single center study, DeVito et al. found that the primary SFT site was the lung/pleura in 28 (34\%) patients, the abdomen/pelvis in 23 (28\%), the extremities in $13(16 \%)$, and the head/neck in $9(11 \%)$. Pathology was benign in $42(51 \%)$ patients and malignant in 40 (49\%) (5). SFTs originating in the pelvis have been reported to be more asymptomatic and more aggressive than SFTs originating in the pleura (12), and about $5-10 \%$ patients with SFT outside the pleura exhibit hypoglycemia $(15,36)$. As shown in Table 2, pelvic SFTs can be classified as bladder SFT, prostate SFT, seminal vesicle SFT, uterine cervix SFT, pelvic SFT, or peritoneum SFT. Table 1 summarizes our systematic review of pelvic SFTs. SFTs can also develop in the bladder, seminal vesicle, cervix, and prostate. For pelvic SFT, tumor resection is the first choice of treatment.

Pelvic SFTs are mostly hypervascular, and thus a lack of preparation for radical resection may cause massive hemorrhaging (3). Therefore, it is important to reduce bleeding during operation. In their study, Soda et al. indicated that pelvic SFTs should be carefully prepared prior to resection (22). Kayani et al. performed successful surgical resection after embolization of the pelvic SFT (12), while Garcia-Amador et al. found that embolization of the median sacral artery and branches of the internal iliac arteries is necessary to facilitate surgery (32). Pelvic tumors, especially pelvic SFTs, are usually supplied by the internal iliac arteries (34). Due to an abundant blood supply and the deep location of tumors, malignant pelvic neoplasms are often difficult to operate on. Transcatheter embolization is an effective treatment for pelvic tumors. In their study, Pisco et al. confirmed the efficacy of preoperative transcatheter embolization for pelvic tumors (37). This was also confirmed in our case: preoperative artery embolization markedly reduced intraoperative hemorrhage, alleviated tumor adhesion, and shortened the operative time. Embolization of internal iliac arteries obviously reduced the operative time and promoted recovery after operation.

There is no standard curative treatment for pelvic SFT other than surgery. Intra-arterial chemotherapy and glucocorticoid therapy are reported to be effective for malignant insulin-like growth factor (IGF-2) secreting SFTs. Furthermore, tyrosine kinase inhibitors, such as imatinib and sunitinib, have also been reported to be effective therapies for unresectable pelvic SFTs (38). It is important to prevent recurrence of pelvic SFT after resection. Therefore, guidelines for the adjuvant therapy of pelvic malignant SFTs are urgently needed in the future.

In conclusion, malignant pelvic SFT is a rare mesenchymal carcinoma. Here, we reported on a patient with a malignant pelvic SFT. The patient indicated the first symptom to be abdominal pain. The patient underwent internal iliac artery embolization in preparation for surgery, and the tumor was completely resected. Finally, the patient recovered well with no recurrence 1 year after transcatheter arterial embolization and surgery.

The take-away concept provided in this case is that transcatheter arterial embolization could be effectively block blood supply of pelvic SFT. After transcatheter arterial embolization, the pelvic SFT is easier to be removed and the got better prognosis. 


\section{Acknowledgments}

Funding: This work was funded by the Youth Foundation of The First Hospital of Jilin University (No. JDYY102019001) and the Science and Technology Development Program of Jilin Province (No. 2020122256JC).

\section{Footnote}

Reporting Checklist: The authors have completed the CARE reporting checklist. Available at https://dx.doi. org/10.21037/tcr-21-887

Conflicts of Interest: All authors have completed the ICMJE uniform disclosure form (available at https://dx.doi. org/10.21037/tcr-21-887). LH reports that this work was supported by Youth Foundation of The First Hospital of Jilin University (No. JDYY102019001) and The Science and Technology Development Program of Jilin Province (No. 2020122256JC). The other authors have no conflicts of interest to declare.

Ethical Statement: The authors are accountable for all aspects of the work in ensuring that questions related to the accuracy or integrity of any part of the work are appropriately investigated and resolved. All procedures performed in studies involving human participants were in accordance with the ethical standards of the ethics committee of the First Hospital of Jilin University and with the Helsinki Declaration (as revised in 2013). Written informed consent was obtained from the patient for publication of this case report and accompanying images. A copy of the written consent is available for review by the editorial office of this journal.

Open Access Statement: This is an Open Access article distributed in accordance with the Creative Commons Attribution-NonCommercial-NoDerivs 4.0 International License (CC BY-NC-ND 4.0), which permits the noncommercial replication and distribution of the article with the strict proviso that no changes or edits are made and the original work is properly cited (including links to both the formal publication through the relevant DOI and the license). See: https://creativecommons.org/licenses/by-nc-nd/4.0/.

\section{References}

1. Sueblinvong T, Judson PL, Downs LS Jr, et al. Solitary fibrous tumors arising from the female pelvis. Obstet Gynecol 2011;118:470-4.

2. Prunty MC, Gaballah A, Ellis L, et al. Solitary Fibrous Tumor of the Pelvis Involving the Urinary Bladder. Urology 2018;117:27-30.

3. Kim MY, Jeon S, Choi SD, et al. A case of solitary fibrous tumor in the pelvis presenting massive hemorrhage during surgery. Obstet Gynecol Sci 2015;58:73-6.

4. Rahimi K, Shaw PA, Chetty R. Solitary fibrous tumor of the uterine cervix. Int J Gynecol Pathol 2010;29:189-92.

5. DeVito N, Henderson E, Han G, et al. Clinical Characteristics and Outcomes for Solitary Fibrous Tumor (SFT): A Single Center Experience. PLoS One 2015;10:e0140362.

6. Kurisaki-Arakawa A, Akaike K, Hara K, et al. A case of dedifferentiated solitary fibrous tumor in the pelvis with TP53 mutation. Virchows Arch 2014;465:615-21.

7. Zhao R, Shan Y, Zou L, et al. Solitary fibrous tumor of the seminal vesicle: A case report. Medicine (Baltimore) 2019;98:e14660.

8. Li XM, Reng J, Zhou P, et al. Solitary fibrous tumors in abdomen and pelvis: imaging characteristics and radiologic-pathologic correlation. World J Gastroenterol 2014;20:5066-73.

9. Fernandez A, Conrad M, Gill RM, et al. Solitary fibrous tumor in the abdomen and pelvis: A case series with radiological findings and treatment recommendations. Clin Imaging 2018;48:48-54.

10. Yang W, Sun F, Liu H, et al. Solitary fibrous tumors of the prostate: A case report. Oncol Lett 2015;10:1617-9.

11. Lu C, Alex D, Benayed R, et al. Solitary fibrous tumor with neuroendocrine and squamous dedifferentiation: a potential diagnostic pitfall. Hum Pathol 2018;77:175-80.

12. Kayani B, Sharma A, Sewell MD, et al. A Review of the Surgical Management of Extrathoracic Solitary Fibrous Tumors. Am J Clin Oncol 2018;41:687-94.

13. Tanaka Y, Nakamoto A, Inada Y, et al. A case of malignant solitary fibrous tumor of the prostatic urethra. BJR Case Rep 2018;4:20180034.

14. England DM, Hochholzer L, McCarthy MJ. Localized benign and malignant fibrous tumors of the pleura. A clinicopathologic review of 223 cases. Am J Surg Pathol 1989;13:640-58.

15. Chen S, Zheng Y, Chen L, et al. A broad ligament solitary fibrous tumor with Doege-Potter syndrome. Medicine (Baltimore) 2018;97:e12564.

16. Nowakowski A, Kozłowski W, Włodarczyk D, et al. A case of a large solitary fibrous tumour of the uterine cervix. 
BMC Womens Health 2014;14:3.

17. Dozier J, Jameel Z, McCain DA, et al. Massive malignant solitary fibrous tumor arising from the bladder serosa: a case report. J Med Case Rep 2015;9:46.

18. Cheng SH, Wang SS, Lee CH, et al. Malignant solitary fibrous tumor of the urinary bladder. J Chin Med Assoc 2012;75:479-82.

19. Heinzelbecker J, Becker F, Pflugmann T, et al. Solitary fibrous tumour of the urinary bladder in a young woman presenting with haemodynamic-relevant gross haematuria. Eur Urol 2008;54:1188-91.

20. Tzelepi V, Zolota V, Batistatou A, et al. Solitary fibrous tumor of the urinary bladder: report of a case with longterm follow-up and review of the literature. Eur Rev Med Pharmacol Sci 2007;11:101-6.

21. Leite KR, Srougi M, Miotto A, et al. Solitary fibrous tumor in bladder wall. Int Braz J Urol 2004;30:406-9.

22. Soda H, Kainuma O, Yamamoto H, et al. Giant intrapelvic solitary fibrous tumor arising from mesorectum. Clin J Gastroenterol 2010;3:136-9.

23. Oguro S, Tanimoto A, Jinzaki M, et al. Imaging findings of solitary fibrous tumor of the prostate: a case report. Magn Reson Imaging 2006;24:673-5.

24. Grasso M, Blanco S, Franzoso F, et al. Solitary fibrous tumor of the prostate. J Urol 2002;168:1100.

25. Funahashi Y, Hattori R, Matsukawa Y, et al. Solitary fibrous tumor of the seminal vesicle. Aktuelle Urol 2010;41:326-7.

26. Boe J, Chimpiri AR, Liu CZ. Solitary fibrous tumor originating in the pelvis: a case report. J Radiol Case Rep 2010;4:21-8.

27. Vossough A, Torigian DA, Zhang PJ, et al. Extrathoracic solitary fibrous tumor of the pelvic peritoneum with central malignant degeneration on CT and MRI. J Magn Reson Imaging 2005;22:684-6.

28. Zhao M, Huang R, He X. A pelvic cellular solitary fibrous tumor with multifocal expression of cytokeratin AE1/AE3. Int J Clin Exp Pathol 2015;8:15437-8.

29. Yan J, Jones RL, Lewis DH, et al. Impact of (18)F-FDG PET/CT imaging in therapeutic decisions for malignant solitary fibrous tumor of the pelvis. Clin Nucl Med 2013;38:453-5.

30. Ando R, Kobayashi D, Naiki T, et al. Pelvic solitary fibrous tumor originally diagnosed as prostatic in origin. Clin Imaging 2012;36:243-5.

31. Tsushimi T, Yagi T, Tomozawa N, et al. Retroperitoneal solitary fibrous tumor of the pelvis with pollakiuria: a case report. BMC Res Notes 2012;5:593.

32. García-Amador C, De La Plaza Llamas R, Ramia Ángel JM, et al. Pelvic Solitary Fibrous Tumor. J Gastrointest Surg 2018;22:1448-9.

33. Kawamura J, Tani M, Kida Y, et al. Successful laparoscopic treatment of a giant solitary fibrous tumor of the mesorectum: A case report and literature review. Asian J Endosc Surg 2017;10:51-4.

34. Katsuno H, Maeda K, Hanai T, et al. Trans-sacral resection of a solitary fibrous tumor in the pelvis: report of a case. Surg Today 2011;41:1548-51.

35. Gao J, Wang X, Yin HF, Jiang Y, Cai YL. Diagnosis and surgical treatment of solitary fibrous tumor of the pelvis. Beijing Da Xue Xue Bao Yi Xue Ban 2013;45:960-4.

36. Wang Y, Wei R, Ji T, et al. Surgical treatment of primary solitary fibrous tumors involving the pelvic ring. PLoS One 2018;13:e0207581.

37. Pisco JM, Martins JM, Correia MG. Internal iliac artery: embolization to control hemorrhage from pelvic neoplasms. Radiology 1989;172:337-9.

38. Ishihara $\mathrm{H}$, Omae $\mathrm{K}$, Iizuka J, et al. Late recurrence of a malignant hypoglycemia-inducing pelvic solitary fibrous tumor secreting high-molecular-weight insulin-like growth factor-II: A case report with protein analysis. Oncol Lett 2016;12:479-84.
Cite this article as: Yan X, Zheng C, Wang J, Li D, Lu J, He L, Wang C. Transcatheter arterial embolization of malignant pelvic solitary fibrous tumor: case report and literature review. Transl Cancer Res 2021;10(11):4979-4987. doi: 10.21037/tcr21-887 